\title{
Evolution of a single defect in an ideal two-dimensional hexagonal soap froth
}

\author{
W. Y. Tam \\ Department of Physics, The Hong Kong University of Science and Technology, Clear Water Bay, Kowloon, Hong Kong
}

(Received 31 July 1998)

\begin{abstract}
We have simulated the evolution of a single defect in an ideal two-dimensional hexagonal soap froth using a physical model based on a combination of mass transfer, vertex movement, and edge relaxation. We find that the defect grows quadratically with time while the mean area of the cells surrounding the defect remains constant in a new scaling state with a topological distribution that differs from the normal froth. Moreover, the number of cell neighbors to the defect is found to grow linearly with time. The results agree with the large- $Q$ Potts model for soap froth and qualitatively with a recent experiment using a bubble raft. [S1063-651X(98)13812-6]

PACS number(s): 82.70.Rr, 02.50.-r
\end{abstract}

Cellular structures observed in such areas as metal grains and biological tissues are common in nature [1]. Soap froth has been a paradigm for the studies of nonequilibrium cellular systems because of its simplicity and relative ease in its experimental setup [2-5]. The system is driven by gas diffusion and in two dimensions the evolution is governed by von Neumann's law:

$$
\frac{d A_{n}}{d t}=\kappa(n-6)
$$

where $A_{n}$ is the area of an $n$-sided cell and $\kappa$ is the diffusion constant. Thus an $n$-sided cell will grow for $n>6$ or shrink for $n<6$. von Neumann's law combined with $T_{1}$ and $T_{2}$ topological transitions led to the coarsening of the froth to a universal scaling state indicated by the stationarity of the distribution functions [5-7]. It is believed that the universal scaling state is independent of initial conditions and has been confirmed by experiments [2-5] and simulations [8-14] starting with disordered initial conditions. However, this common belief has been tested by a topological simulation for the evolution of an ideal two-dimensional hexagonal froth with a singe defect [15]. This study suggested that with this particular initial condition the system will evolve to a new scaling state different from the usual scaling state of normal froth. The result was reexamined by recent simulations of the large- $Q$ Potts model [16] and direct simulation [17] using the method of Weaire and Kermode [11]. Both the large- $Q$ Potts model [18] and the direct simulation [11] have been shown to reproduce well the evolution of twodimensional soap froth. These studies support the previous suggestion qualitatively. However, it is not clear whether the new scaling state has been reached due to the small sample size in the direct simulation [17] and lattice effects in the Potts model [16]. Previous experiments on dry twodimensional soap froth have shown a transient that differs from the normal scaling state for relatively ordered froth with many topological defects [19]. However, the system eventually reached the normal scaling state at long time. A recent experimental study of a single topological defect in a two-dimensional bubble raft [20] provides some insight into the new scaling state despite the fact that the experiment corresponds to wet froth. Nevertheless, this study reenforces the need to better quantify the new scaling state. While an experiment starting with a single defect in an ideal dry hexagonal froth is highly desirable, we use the direct simulation approach to obtain the long time behavior for the defect and the cluster of cells surrounding the defect.

The direct simulation used in this study has been reported recently in detail $[13,14]$ and is similar to that of Weaire and Kermode (WK) [11] and Aref and Herdtle (AF) [12]. The simulation is based on a physical model where the movement of cell walls and vertices due to gas diffusion through the cell walls between neighboring cells is followed by finding the quasiequilibrium configuration such that three walls (edges) meet at $120^{\circ}$ and the pressure difference across each edge is balanced by the tensional force acting on the edge after each "diffusion step." Furthermore, $T 1$ and $T 2$ topological transitions are implemented with a series of criteria to eliminate spurious behavior and enable tight control on the accuracy (see Refs. [13,14] for detailed implementation). Note that, unlike the simulations of WK [11] and AF [12], von Neumann's law is not built into the model but is well satisfied for every individual $n$-sided cell [13]. The model has been shown to reproduce quantitatively the evolution of two-dimensional soap froth $[13,14]$ and has also been applied to a recent study of ancestral cells (ancestors) that survive for long times [21].

The simulation starts with a perfect two-dimensional hexagonal lattice with 5000 cells. The defect is created by suppressing an edge in the lattice giving an eight-sided cell with two symmetrical five-sided cells and six hexagonal cells surrounding the defect, as shown in Fig. 1(a). This initial condition is the same as that used in Ref. [17]. Using periodic boundary conditions, the froth is allowed to evolve according to the dynamics as stated above for normal soap froth. The defect is found to grow while the surrounding cells shrink, leading to a circular propagating front "consuming" the regular hexagonal lattice, as shown in Fig. 1. The result is similar to the large- $Q$ Potts model except that the Potts model gives a less symmetric front probably due to lattice effects.

Figure 2 shows qualitatively a quadratic dependence of the normalized area $a_{\mathrm{def}}=A_{\mathrm{def}} / A_{0}$ of the defect as a function of the simulation time $t$. Here $A_{\text {def }}$ is the area of the defect and $A_{0}$ is the lattice size. A power-law fit for $a_{\text {def }}$ versus $t$ 

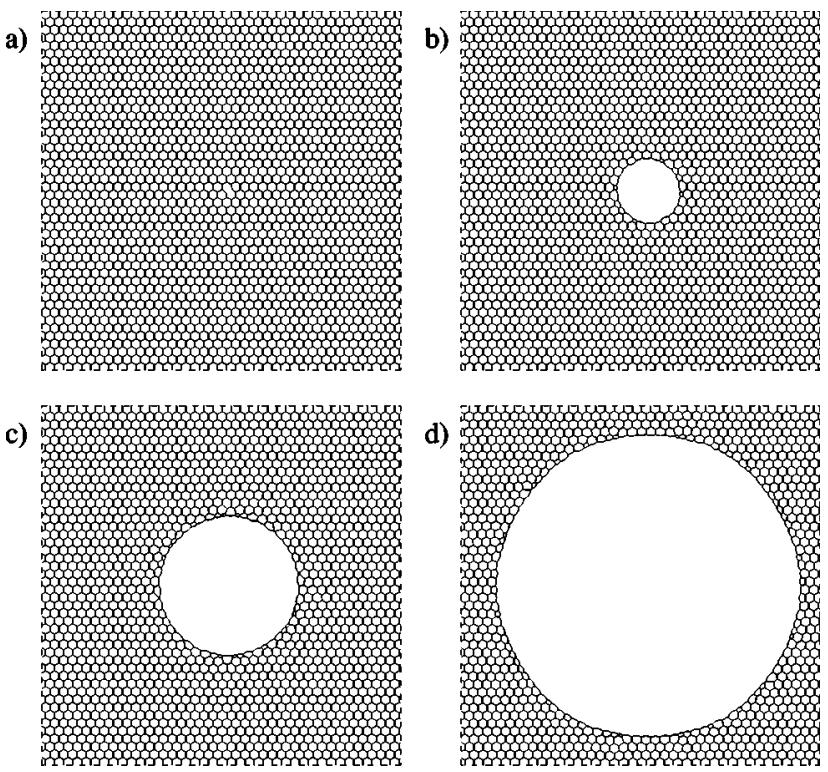

FIG. 1. Evolution of a single defect in a perfect hexagonal froth at (a) 0.00 , (b) 9.81 , (c) 19.80, and (d) 39.80 simulation time units.

gives an exponent of $2.20 \pm 0.01$. The result is the same as the previous simulation with about 1000 cells where the radius of the defect increases roughly linearly with time $[13,14]$. Since only the cells around the defect are affected, it is desirable to define a cluster $\{c\}$ as cells having at least one neighbor that is not six-sided [15]. However, the cluster is heavily dominated by the defect when the defect is much bigger than the lattice size. Thus an alternate choice is to exclude the defect to form a boundary cluster $\left\{c^{\prime}\right\}$ surrounding the defect [20]. In addition, to facilitate the use of another time scale, the cells $\{b\}$ that are neighbors to the defect will also be monitored. Figure 2(b) shows the mean area (normalized by the hexagonal lattice) of the cluster $\{c\}$, the boundary cluster $\left\{c^{\prime}\right\}$, and the neighbor cells $\{b\}$ as a function of time. The mean area of the cluster $\left\langle a_{c}\right\rangle$ is found to increase almost linearly due to the effect of the defect. In contrast, the mean areas of the boundary cluster $\left\langle a_{c^{\prime}}\right\rangle$ and the neighbor cells $\left\langle a_{b}\right\rangle$ approach stationary values of 0.83 \pm 0.03 and $0.69 \pm 0.01$, respectively. The steady-state value of $\left\langle a_{c^{\prime}}\right\rangle$ is between those of the Potts-model simulation $\left(\left\langle a_{c^{\prime}}\right\rangle=0.88 \pm 0.08[16]\right)$ and the bubble-raft experiment $\left(\left\langle a_{c^{\prime}}\right\rangle=0.77 \pm 0.04[20]\right)$.

Figure 3(a) shows an almost linear dependence on the number of cells $N_{b}$ that are neighbors to the defect with the simulation time. A power-law fit of $N_{b}$ versus $t$ gives an exponent of $1.09 \pm 0.01$. Hence if $N_{b}$ is used as a new time scale, the area of the defect should grow quadratically with $N_{b}$. Indeed, a power-law fit of $a_{\text {def }}$ versus $N_{b}$ gives an exponent of $2.01 \pm 0.01$. Furthermore, it is expected that the number of cells $N_{c^{\prime}}$ in the boundary cluster $\left\{c^{\prime}\right\}$ is proportional to $N_{b}$, as shown in Fig. 3(b). A power-law fit of $N_{c^{\prime}}$ versus $N_{b}$ gives an exponent of $0.99 \pm 0.02$. In addition, the ratio $N_{c^{\prime}} / N_{b}$ is found to be $1.80 \pm 0.02$. Physically, the boundary cluster $\left\{c^{\prime}\right\}$ consists of one layer of neighbor cells $\{b\}$ surrounding the defect plus a partial layer $(80 \%)$ of sixsided cells next to the neighbor cells. The mean area of the cells in the partial layer differs only by $1 \%$ from the regular lattice, indicating that they are not affected by the defect very
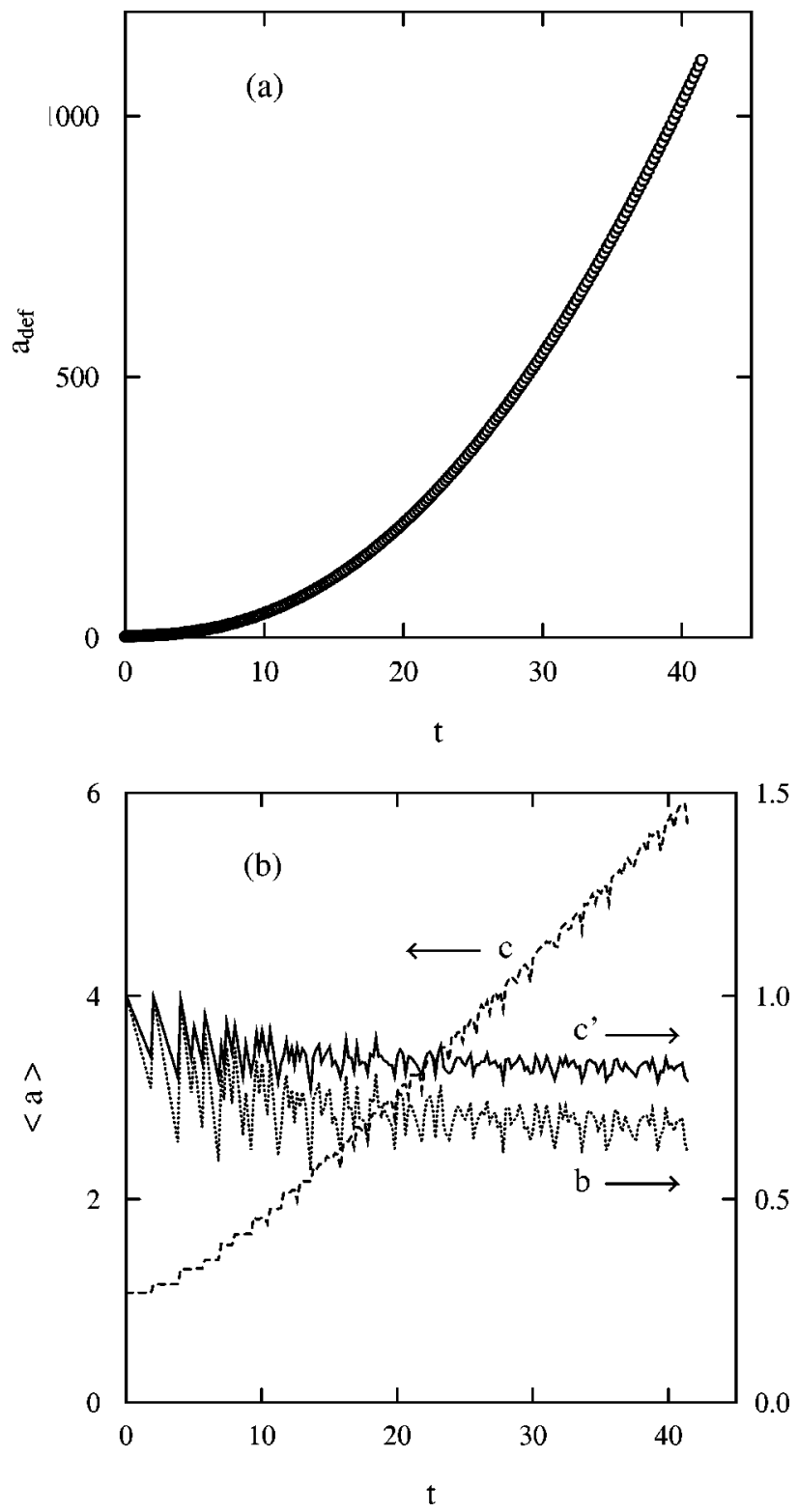

FIG. 2. (a) The normalized area of the defect as a function of simulation time. (b) The normalized mean number of the cluster $\{c\}$ (dashed line), the boundary cluster $\left\{c^{\prime}\right\}$ (solid line), and the neighbor cells $\{b\}$ (dotted line) as a function of simulation time.

much. This implies that the propagation of the defect has a relatively "sharp" interface such that there is a thin boundary, at most two layers of cells, between the defect and the regular lattice. The above result confirms the topological simulation [15] in which the number of "killed" cells was used as the time scale because the number of "killed" cells scales with the defect area.

Figure 4(a) shows the second moment of the topological distribution $\rho(n)$ defined as $\mu_{2}=\Sigma(n-6)^{2} \rho(n)$ for the cluster $\{c\}$, the boundary cluster $\left\{c^{\prime}\right\}$, and the neighbor cells $\{b\}$ as a function of time. It is clear that $\mu_{2}$ for the cluster $\{c\}$ increases with time primarily due to the increase in the number of sides of the defect and is in agreement with the Potts simulation [16]. However, $\mu_{2}$ for both the boundary cluster $\left\{c^{\prime}\right\}$ and the neighbor cells $\{b\}$ reaches steady values of $0.76 \pm 0.01$ and $1.37 \pm 0.02$, respectively. In addition, the to- 

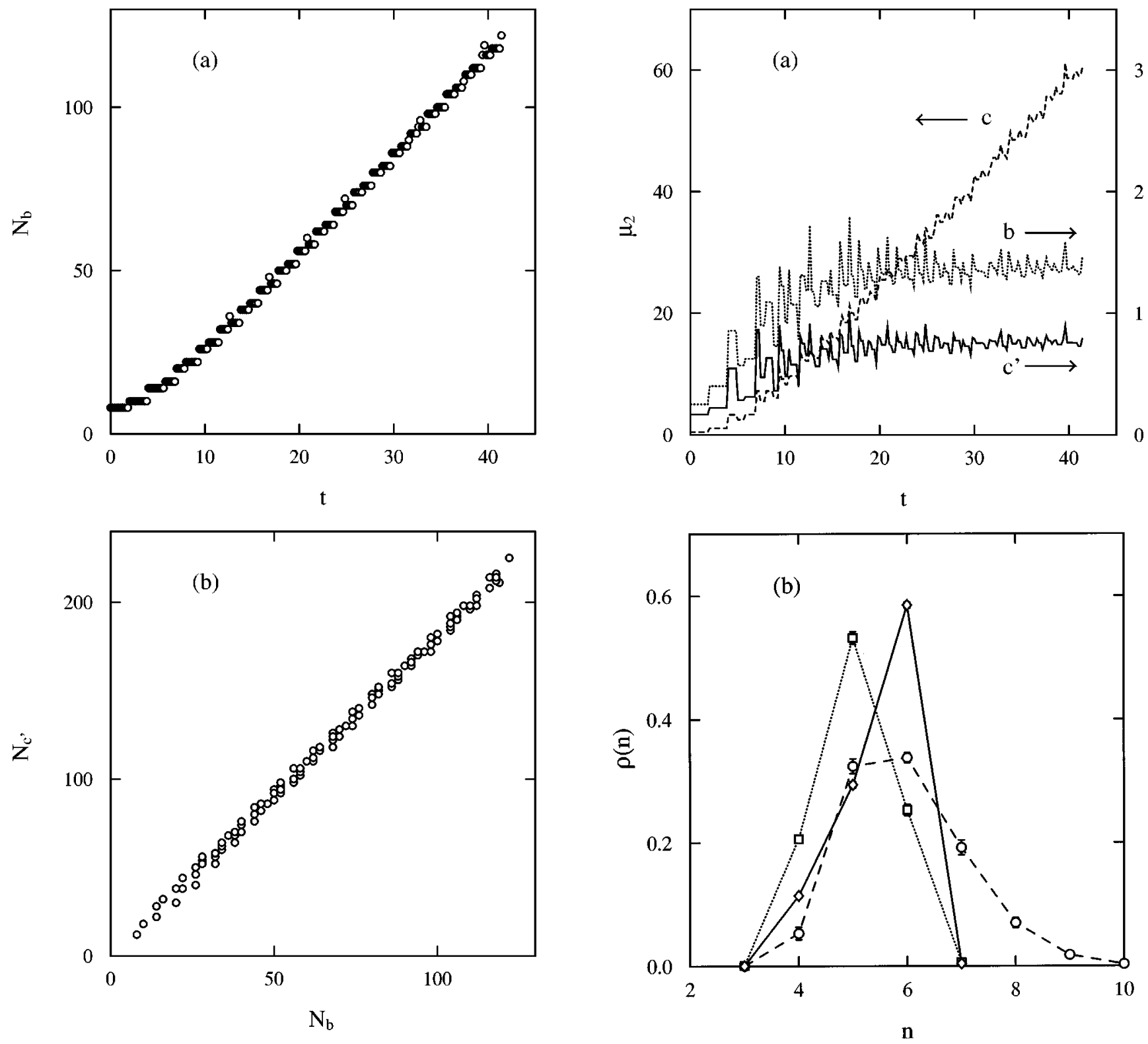

FIG. 3. (a) The number of cell neighbors to the defect as a function of simulation time. (b) The number of cells in the boundary cluster $\left\{c^{\prime}\right\}$ versus the number of neighbor cells.

pological distributions $\rho(n)$ for both sets of cells $\left\{c^{\prime}\right\}$ and $\{b\}$ are found to be stationary and the mean values are shown in Fig. 4(b) using data with $N_{b}$ larger than 80. The above results strongly support a nontrivial new scaling state that is different from the normal froth shown as a dashed line in Fig. 4(b). The $\mu_{2}$ and the topological distribution for the boundary cluster $\left\{c^{\prime}\right\}$ agree well with the Potts simulation [16] despite the fact that there is still a slow increase in $\mu_{2}$ shown in Fig. 3 of Ref. [16]. One striking feature of the topological distribution of the boundary cluster $\left\{c^{\prime}\right\}$ and the neighbor cells $\{b\}$ is that there is no cell with eight sides or more, indicating that they are relatively more "ordered" than the normal froth.

In conclusion, we have simulated the evolution of a single defect in a perfect hexagonal froth using a physical model and we have shown the existence of a new scaling state for the cells surrounding the defect. The results are in good agreement with previous topological and large- $Q$ Potts simu-

FIG. 4. (a) The second moment of the topological distribution for the cluster $\{c\}$ (dashed line), the boundary cluster $\left\{c^{\prime}\right\}$ (solid line), and the neighbor cells $\{b\}$ (dotted line) as a function of simulation time. (b) The topological distribution for the boundary cluster $\left\{c^{\prime}\right\}$ (solid line), the neighbor cells $\{b\}$ (dotted line), and the normal froth (dashed line).

lations $[15,16]$. However, the comparison with the experiment [20] using a bubble raft is only qualitative due to the fact that the experiment has a short duration and is a wet froth while the direct simulation is assumed to be fluidless in the soap films. Thus an experiment with a single defect in a dry hexagonal froth is highly desirable.

\section{ACKNOWLEDGMENTS}

W. Y. Tam acknowledges support from Direct Allocation Grant No. 96/97.SC22 of the Hong Kong University of Science and Technology. Valuable discussions with K. Y. Szeto are also acknowledged. The dynamic code for the simulation was obtained from J. J. Chae and M. Tabor. 
[1] D. Weaire and N. Rivier, Contemp. Phys. 25, 59 (1984).

[2] J. A. Glazier, S. Gross, and J. Stavans, Phys. Rev. A 36, 306 (1987).

[3] J. A. Glazier, M. P. Anderson, and G. S. Grest, Philos. Mag. B 62, 6115 (1990).

[4] E. A. Holm, J. A. Glazier, D. J. Srolovitz, and G. S. Grest, Phys. Rev. A 43, 2662 (1991).

[5] J. Stavans, Rep. Prog. Phys. 56, 733 (1993).

[6] H. Flyvbjerg, Phys. Rev. E 47, 4037 (1993); Physica A 194, 298 (1993).

[7] J. Stavans, E. Domany, and D. Mukamel, Europhys. Lett. 15, 479 (1991).

[8] B. Levitan, E. Slepyan, O. Krichevshy, J. Stavans, and E. Domany, Phys. Rev. Lett. 173, 756 (1994).

[9] B. Levitan and E. Domany, Phys. Rev. E 54, 2766 (1996).

[10] B. Levitan and E. Domany, Int. J. Mod. Phys. B 10, 3765 (1996).

[11] D. Weaire and J. Kermode, Philos. Mag. B 48, 245 (1983); 47, L29 (1983); 48, 379 (1984); J. Kermode and D. Weaire, Comput. Phys. Commun. 60, 75 (1990).
[12] H. Aref and T. Herdtle, in Topological Fluid Mechanics, edited by $\mathrm{H}$. Moffatt and A. Tsinober (Cambridge University Press, Cambridge, England, 1990), pp. 745-764; T. Herdtle and A. Aref, J. Fluid Mech. 241, 233 (1992).

[13] J. J. Chae, Ph.D. thesis, University of Arizona, 1995 (unpublished).

[14] J. J. Chae and M. Tabor, Phys. Rev. E 55, 598 (1996).

[15] B. Levitan, Phys. Rev. Lett. 72, 4057 (1994).

[16] Y. Jiang, J. C. M. Mombach, and J. A. Glazier, Phys. Rev. E 52, R3333 (1995).

[17] H. J. Ruskin and Y. Feng, J. Phys.: Condens. Matter 7, L553 (1995).

[18] J. A. Glazier, M. P. Anderson, and G. S. Grest, Philos. Mag. B 62, 615 (1990).

[19] J. Stavans and J. A. Glazier, Phys. Rev. Lett. 62, 1318 (1989); Phys. Rev. A 42, 5049 (1990).

[20] A. Abd el Kader and J. C. Earshaw, Phys. Rev. E 56, 3251 (1997).

[21] W. Y. Tam, K. M. Cheung, and K. Y. Szeto, Phys. Rev. E 57, 7354 (1997). 